\title{
Effect of Survivin gene therapy via lentivirus vector on the course of intervertebral disc degeneration in an in vivo rabbit model
}

\author{
BIN YUE* ${ }^{*}$ YAZHOU LIN*, XUEXIAO MA, GUOQING ZHANG and BOHUA CHEN \\ Department of Orthopedic Surgery, The Affiliated Hospital of Qingdao University, Qingdao, Shandong 266003, P.R. China
}

Received September 30, 2015; Accepted October 3, 2016

DOI: $10.3892 / \mathrm{mmr} .2016 .5830$

\begin{abstract}
The aim of the current study was to use gene therapy to attenuate or reverse the degenerative process within the intervertabral disc. The effect of survivin gene therapy via lentiviral vector transfection on the course of intervertebral disc degeneration was investigated in the current study in an in vivo rabbit model. A total of 15 skeletally mature female New Zealand White rabbits were randomly divided into three groups: Punctured blank control group (group A, n=5), punctured empty vector control group (group $\mathrm{B}, \mathrm{n}=5$ ) and the treatment group (group $\mathrm{C}, \mathrm{n}=5$ ). Computed tomography-guided puncture was performed at the L3-L4 and L4-L5 discs, in accordance with a previously validated rabbit annulotomy model for intervertebral disc degeneration. After 3 weeks, a lentiviral vector (LV) carrying survivin was injected into the nucleus pulposus. The results demonstrated that through magnetic resonance imaging, histology, gene expression, protein content and apoptosis analyses, group A and B were observed to exhibit disc degeneration, which increased over time, and no significant difference was observed between the two groups $(\mathrm{P}>0.05)$. However, there was reduced disc degeneration in group $\mathrm{C}$ compared with the punctured control groups, and the difference was statistically significant $(\mathrm{P}<0.05)$. Overall, the results of the present study demonstrated that injection of the LV carrying survivin into punctured rabbit intervertebral discs acted to delay changes associated with the degeneration of the discs. Although data from animal models should be extrapolated to the human condition with caution, the present study suggests potential for the use of gene therapy to decelerate disc degeneration.
\end{abstract}

Correspondence to: Dr Bohua Chen, Department of Orthopedic Surgery, The Affiliated Hospital of Qingdao University, 16 Jiangsu Road, East, Qingdao, Shandong 266003, P.R. China E-mail: bhchen@hotmail.com

*Contributed equally

Key words: genetic therapy, survivin, intervertebral disc degeneration, animal experimentation, magnetic resonance imaging, molecular biology, anatomy \& histology

\section{Introduction}

Approximately $80 \%$ of the aging population suffer from lower back pain at some point in their lives (1-3). Back pain associated with intervertebral disc degeneration (IDD) clearly affects quality of life and work productivity, and significantly impacts health care spending (4). However, the currently used first-line treatments, including activity modification, analgesic and anti-inflammatory medications, physical therapy and surgery only relieve symptoms temporarily and do not target repair or deceleration of the underlying degenerative progress $(5,6)$. Alternative and less invasive treatment methods such as gene therapy are being developed, aiming to slow or reverse the degenerative process of IDD.

Developing approaches to stimulating regeneration of disc tissue or slowing the degeneration progression requires an understanding of the causes of the degeneration. Previous studies have demonstrated that the number of nucleus pulposus (NP) cells reduces and the composition of the extracellular matrix associated with these cells is altered in degenerative discs (7-9). In vitro and in vivo studies have suggested that the cellular loss attributed to the excessive apoptosis of disc cells serves an important role in disc degeneration $(10,11)$. These mechanisms provide direction and fundamental information for gene therapy.

Survivin protein, a baculoviral inhibitor of apoptosis, serves an important role in the regulation of mitosis progression and apoptosis inhibition (12). In osteoarthritis and rheumatoid arthritis, survivin stimulates chondrocyte proliferation and inhibits apoptosis $(13,14)$. Furthermore, preliminary studies have indicated that survivin is expressed in fetal disc tissue, and that there is a differential expression of survivin between degenerated NP tissue and comparatively normal NP tissue $(15,16)$. In a previous study, it was demonstrated that survivin is re-expressed in disc degeneration disease and is required for degenerated NP cell proliferation and anti-apoptosis in vitro (17). Thus, it is suggested that the use of survivin as a target gene for gene therapy for IDD may be efficacious. However, to the best of our knowledge, at present no studies investigating gene therapy with survivin via a lentiviral vector (LV) and the course of intervertebral disc degeneration in vivo have been conducted.

The present study investigated the use of gene therapy in improving IDD by administering LV-survivin into disc cells. This validated IDD model was used to determine whether 
treatment with LV-survivin can ameliorate the course of NP cell apoptosis. The results provided information for gene therapy to decelerate IDD in future clinical application.

\section{Materials and methods}

Materials. A total of 15 skeletally mature female New Zealand White rabbits (3-months-old, weighing 2-2.5 kg; Chongqing Kangda Juxin Rabbit Co., Ltd., Qingdao, China) were randomly divided into three groups: Group A, punctured blank control group (degeneration and placebo injection, $\mathrm{n}=5$ ); group B, punctured empty vector control group (degeneration and an empty LV injection, $n=5$ ); and group $C$, treatment group (degeneration and LV-survivin injection, $\mathrm{n}=5$ ). $\mathrm{LV}$-survivin and empty LV were purchased from Genechem Co., Ltd. (Shanghai, China). The titers of LV-survivin and empty LV were $2 \times 10^{8}$ and $1 \times 10^{9} \mathrm{TU} / \mathrm{ml}$, respectively. The Animal Care and Use Committee of the Affiliated Hospital of Qingdao University (Shandong, China), approved all animal experimental protocols, which followed the principles expressed in the National Institute of Health Guide. The rabbits were maintained separately at room temperature with $16: 8 \mathrm{~h}$ light:dark cycle, and were fed with rabbit feed (Qingdao Kangda Foodstuffs Co., Ltd., Qingdao, China).

Puncture surgery. Computed tomography (CT)-guided percutaneous needle puncture technology was used to establish this model (18). Magnetic resonance imaging (MRI) and histology highlighted alterations that resembled the hallmarks of human disc degeneration. In brief, rabbits were anesthetized with Su Mian Xin II $(0.15 \mathrm{ml} / \mathrm{kg}$ intramuscularly; Kangda Medical Products Co., Ltd., Shanghai, China) and atropine sulfate $(0.05 \mathrm{mg} / \mathrm{kg}$ intramuscularly; Jiangsu Lianshui Pharmaceutical Co., Ltd., Lianshui, China), the position of the target discs (L3-L4 and L4-/L5) were identified using $\mathrm{CT}$, and then a 16-gauge needle was inserted toward the center of the disc, with CT guidance. The needle pinpoint was confirmed to be positioned in the disc center by CT (Fig. 1). Postoperatively, the rabbits were housed in individual cages and injected intramuscularly with penicillin at $80 \times 10^{5} \mathrm{U}$ to prevent infection.

Injection surgery. At 3 weeks post-puncture surgery, groups A, $\mathrm{B}$ and $\mathrm{C}$ were injected with equivalent phosphate-buffered saline (PBS; $50 \mu 1)$, empty LV (5x10 $\% / 50 \mu 1$; Genechem Co., Ltd., Shanghai, China), and LV-survivin $\left(5 \times 10^{6} / 50 \mu 1\right.$; Genechem Co., Ltd.), respectively, using CT-guided percutaneous needle injection technology. This time point was selected due to the fact that it is the earliest that MRI could identify changes of degeneration with this model (18). An $100 \mu 1$ microinjector (Hamilton Bonaduz AG, Bonaduz, Switzerland) was used for therapeutic injections into the center of the NP.

MRI. MRIs were obtained at time-point 0 (prior to puncture), 3 weeks post-puncture (prior to injection), and 12 weeks post-puncture (prior to sacrifice) for all rabbits. A 3-T standard human knee coil was used to obtain T2-weighted images (repetition time $=2120 \mathrm{~ms}$, echo time $=113 \mathrm{~ms}$, slice thickness $=0.6 \mathrm{~mm}$ ). The rabbits were anesthetized and placed in the knee coil in the supine position.
Table I. Details of nucleotide sequences of sense and antisense primers, RT-qPCR amplification product.

\begin{tabular}{|c|c|c|}
\hline Gene & Primer & $\begin{array}{l}\text { PCR } \\
\text { product } \\
\text { (bp) }\end{array}$ \\
\hline Survivin & $\begin{array}{l}\text { F: CAGATGACGACCCCATAGAGGA } \\
\text { R: CCTTTGCAATTTTGTTCTTGGC }\end{array}$ & $141 \mathrm{bp}$ \\
\hline GAPDH & $\begin{array}{l}\text { F: GGATTTGGTCGTATTGGG } \\
\text { R: GGAAGATGGTGATGGGATT }\end{array}$ & $205 \mathrm{bp}$ \\
\hline
\end{tabular}

RT-qPCR, reverse transcription-quantitative polymerase chain reaction; bp, base pairs.

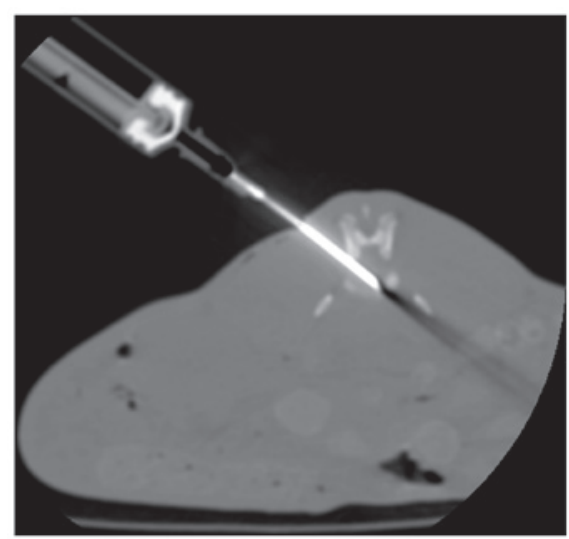

Figure 1. Computed tomography-guided percutaneous needle puncture technology.

Histological processing. All rabbits were sacrificed by air injection into the ear vein 12 weeks subsequent to the initial puncture surgery, following the final MRI scan. Immediately after sacrifice, spines were dissected out en bloc. The L3-L4 discs ( $n=5$ per group) were prepared for histology. Two discs from each group were fixed and then dehydrated using a histology tissue processor and embedded in paraffin. Next, the discs were sectioned at a thickness of $5 \mu \mathrm{m}$ in the coronal plane. The sections were stained with hematoxylin and eosin (H\&E; Beijing Leagene Biotechnology Co., Ltd., Beijing, China). The other discs were embedded in Tissue-Tek ${ }^{\mathrm{TM}}$ (Sakura, CA, USA) and then sectioned at a thickness of $5 \mu \mathrm{m}$ in the coronal plane in a freezing microtome (Leica CM1950; Leica Microsystems GmbH, Wetzlar, Germany). The sections were stained for terminal deoxynucleotidyl transferase dUTP nick end labeling (TUNEL; Nanjing KeyGEN Biotech Co., Ltd., Nanjing, China) and were imaged using an Olympus BX51 microscope (Olympus Corporation, Tokyo, Japan).

Gene expression and protein content of survivin. A total of 3 L4-L5 discs from each group ( $\mathrm{n}=5$ per group) were prepared for reverse transcription-quantitative polymerase chain reaction (RT-qPCR) and western blot analysis. mRNA was extracted from NP tissue using TRIzol ${ }^{\mathrm{TM}}$ (Invitrogen; Thermo Fisher Scientific, Inc., Waltham, MA, USA) and the absorbance at was measured at 260 and $280 \mathrm{~nm}$ for 


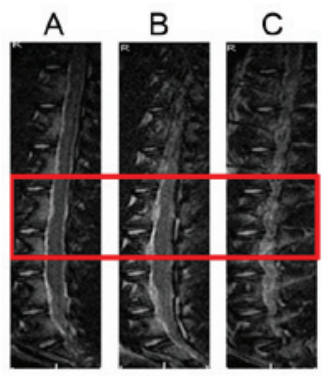

0 Week

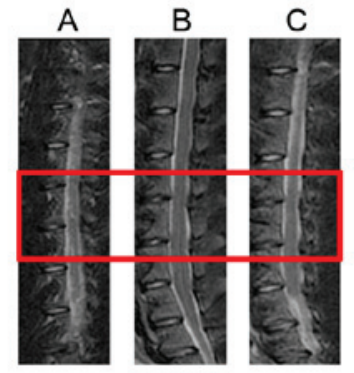

3 Week

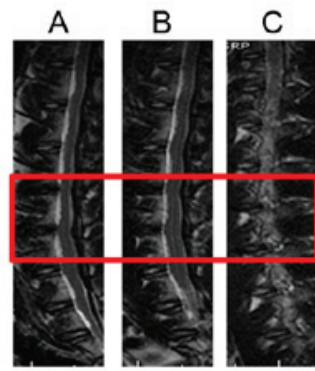

12 Week

Figure 2. T2-weighted midsagittal magnetic resonance imaging of different groups at different time points. A, punctured blank control group; B, punctured empty vector control group; $\mathrm{C}$, treatment group.

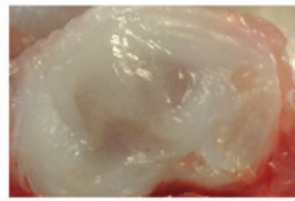

Normal

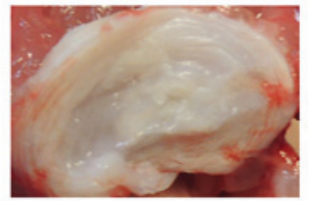

A

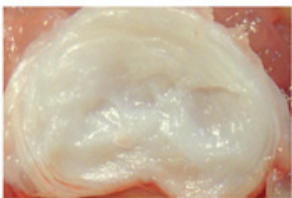

B

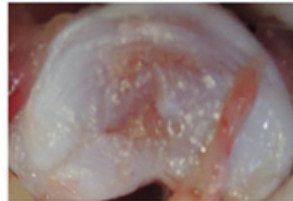

C

Figure 3. Disc morphology of the different groups. A, punctured blank control group; B, punctured empty vector control group; C, treatment group.

quantification and quality control. A total of $1 \mu \mathrm{g}$ mRNA was reverse transcribed to cDNA using PrimeScript ${ }^{\mathrm{TM}}$ RT reagent kit (cat no. DRR037A; Takara Bio, Inc., Otsu, Japan), and the reaction product was treated with RNAse-free DNase I. PCR was conducted using the cycling conditions (LightCycler 480 Instrument II) as stated by the manufacturer's instructions. Primers were designed and purchased from Shanghai Sangon Biotech Co., Ltd. (Shanghai, China) (Table I). Another specific primer pair for human GAPDH was used as an internal control. In each experiment, samples were analyzed in duplicate. The normalized target gene expression was determined through the comparative Cq method ( $\Delta \Delta \mathrm{Cq}$ method) (19).

NP tissues were washed with ice-cold PBS, weighed and then ground using a pestle and mortar. Lysis was performed on ice for $45 \mathrm{~min}$ using $200 \mu \mathrm{l}$ radioimmunoprecipitation assay buffer with $2 \mu \mathrm{l}$ phenylmethanesulfonylfluoride for every $100 \mu \mathrm{g}$ of tissue. Subsequently, the lysis solution was centrifuged at $5,700 \times g$ at $4^{\circ} \mathrm{C}$ for $20 \mathrm{~min}$. For the western blot analysis of survivin, GAPDH was used as an internal control, and proteins were resolved through sodium dodecyl sulfate-polyacrylamide gel electrophoresis using a $10 \%$ gel, prior to transferring onto Immobilon P membranes (EMD Millipore, Billerica, MA, USA). The membranes were blocked with $5 \%$ fat-free dried milk and probed with a monoclonal rabbit anti-survivin antibody (1:1,000; ab76424; Abcam, Cambridge, MA) and anti-GAPDH antibody $\left(1: 2,000\right.$; ab9485; Abcam) for $8 \mathrm{~h}$ at $4^{\circ} \mathrm{C}$. Subsequent to incubation with horseradish peroxidase-conjugated secondary antibodies (1:2,000; CW0103; Beijing Kangwei Century Biotechnology Co., Ltd., Beijing, China) for $4 \mathrm{~h}$ at room temperature, the positive bands were visualized using chemiluminescence (Pierce Biotechnology, Inc., Rockford, IL, USA).

Cell culture and caspase-3 activity assay. The remaining L4-L5 discs were prepared for measuring caspase-3 activity. NP cells were cultured as described in a previous study (20). In brief, the NP tissue was cut into small pieces $\left(\sim 1 \mathrm{~mm}^{2}\right)$ and then digested with $0.25 \%$ trypsinase $\left(\mathrm{HyClone}^{\mathrm{TM}}\right.$; GE Healthcare Life Sciences, Logan, UT, USA) at $37^{\circ} \mathrm{C}$ under gentle agitation for $20 \mathrm{~min}$. Subsequently, $0.5 \%$ collagenase type II (MP Biomedicals, LLC, Santa Ana, CA, USA) was used at $37^{\circ} \mathrm{C}$ for approximately $4 \mathrm{~h}$. The cells were transferred to a $12.5 \mathrm{~cm}^{2}$ culture flask at a density of $10^{5}$ cells $/ \mathrm{cm}^{2}$. The cells were cultured in a $\mathrm{CO}_{2}$ incubator (SANYO Electric Co., Ltd., Osaka, Japan) at $37^{\circ} \mathrm{C}$ with humidity, and grown in Dulbecco's modified Eagle's medium (DMEM)-F12 containing 15\% fetal calf serum. The experiments were analyzed in duplicate. For ischemic conditions, NP cells were cultured in DMEM culture medium (glucose deprivation) in a $\mathrm{CO}_{2}$ incubator at $37^{\circ} \mathrm{C}$ with $1 \%$ oxygen and $95 \%$ humidity. These cells were subsequently used for analyzing caspase- 3 activity. The caspase- 3 activity was measured using the Caspase-3 Colorimetric Assay kit (BioVision, Inc., Milpitas, CA, USA). The NP cells were counted and pelleted at $1.5 \times 10^{6}$ cells. The cells were then re-suspended in cell lysis buffer, and $50 \mu \mathrm{l} 2 \mathrm{X}$ reaction buffer (containing $10 \mathrm{mM}$ DTT) and $5 \mu \mathrm{l}$ of DEVE-pNA were added. The samples were incubated for $90 \mathrm{~min}$ at $37^{\circ} \mathrm{C}$ and their optical densities were read at $405 \mathrm{~nm}$ using a microtiter plate reader (Sunrise ${ }^{\mathrm{TM}}$; Tecan Group, Ltd., Männedorf, Switzerland).

Statistical analysis. All values were presented as the mean \pm standard error. Student's t-test and one-way analysis of variance with a post hoc Fisher's least significant difference test were applied to measure the statistical significance of the differences. Statistical analyses were performed using SPSS software for Windows (version 19; SPSS, Inc., Chicago, IL, USA).

\section{Results}

Imaging. The T2-weighted midsagittal MRI scans of groups A and $\mathrm{B}$ were darkened and reduced in area from 0-12 weeks, consistent with the degeneration. Group $\mathrm{C}$ demonstrated 


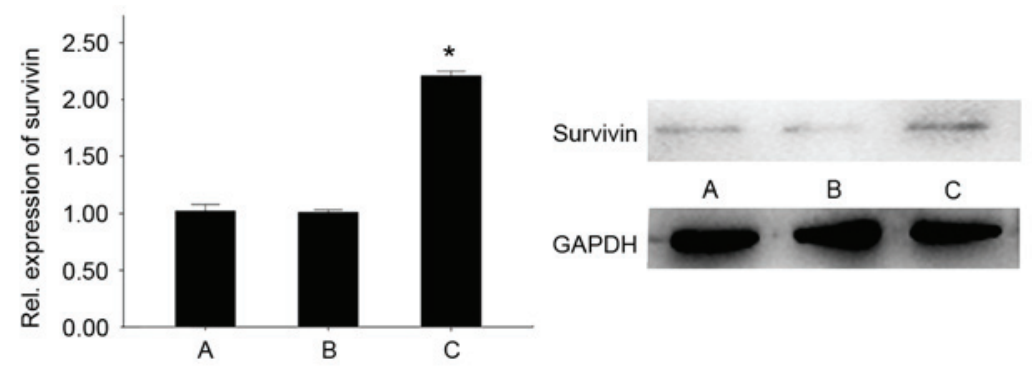

Figure 4. Gene expression and protein content of survivin. "P<0.05 vs. group A and B. A, punctured blank control group; B, punctured empty vector control group; C, treatment group.

A

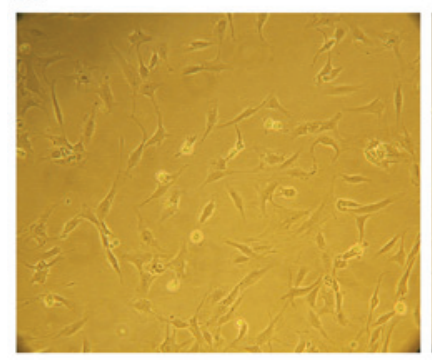

B

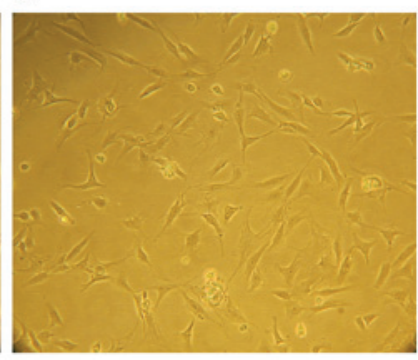

C

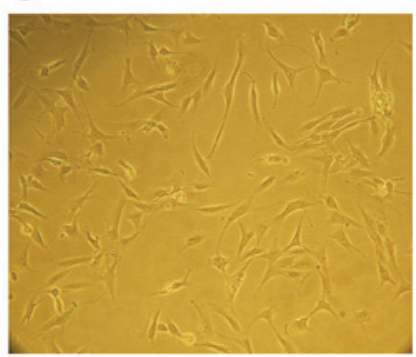

Figure 5. Morphology of nucleus pulposus cells in the different groups (magnification, x100). A, punctured blank control group; B, punctured empty vector control group; C, treatment group.

reduced qualitative evidence of degeneration when compared with groups A and B; treated NPs did not darken and reduce in area as much as the punctured discs (Fig. 2). The morphology of normal discs indicated an abundance of water and ECM. Discs from groups A and B were reduced in the quantity of both water and ECM, and exhibited fibrotic morphology, consistent with the degree of degeneration. However, the quantity of water and ECM in group $C$ were between that of the normal group (group A) and the punctured group (group B) (Fig. 3).

Gene expression and protein content of survivin. The gene and protein content of each group were analyzed by RT-qPCR and western blot analysis. Gene expression and protein content of survivin in group $\mathrm{C}$ demonstrated a significant increase compared with groups $\mathrm{A}$ and $\mathrm{B}(\mathrm{P}<0.05)$. No significant difference was observed between groups A and B (Fig. 4).

The morphology of NP cells. The NP cells were attached to the culture dish after 3-5 days of culture. The cell morphology gradually elongated and became triangular or polygonal, and the cytoplasm became plump and equally distributed, and the attached cells exponentially increased. Subsequently, 10-15 days later, $90 \%$ of the cells formed colonies. No significant differences of cell morphology were observed among the three groups (Fig. 5).

Caspase-3 activity reflected apoptosis of NP cells. The caspase-3 activity of NP cells was assayed. Under the normal culture conditions, group $\mathrm{C}$ did not exhibit significantly altered caspase- 3 activity compared with groups $\mathrm{A}$ and $\mathrm{B}(\mathrm{P}=0.082$ and $\mathrm{P}=0.539$, respectively). When exposed to ischemia in vitro ( $1 \%$ oxygen, glucose deprivation), the caspase-3 activity of all

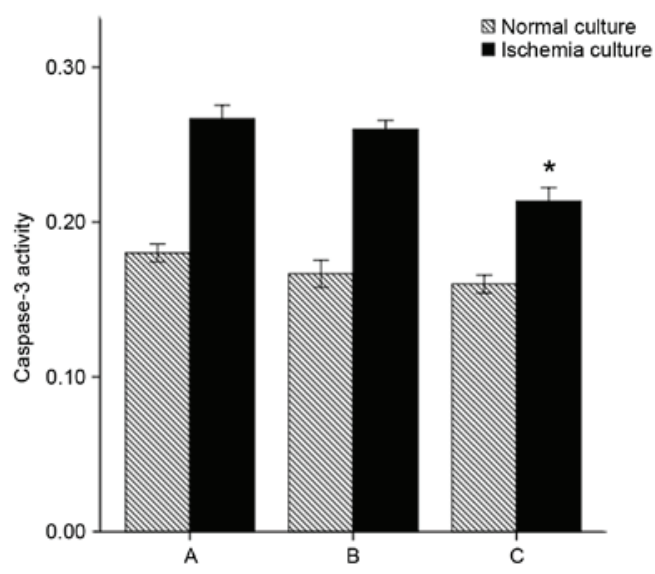

Figure 6. Caspase-3 activity reflected the apoptosis of nucleus pulposus cells ${ }^{*} \mathrm{P}<0.05$ vs. group A and B. A, punctured blank control group; B, punctured empty vector control group; $\mathrm{C}$, treatment group.

groups increased compared with the normal culture conditions $(\mathrm{P}=0.00)$. However, caspase- 3 activity remained significantly reduced overall for group $\mathrm{C}$ (group $\mathrm{C}+$ in vitro ischemia vs. group $\mathrm{A}+$ in vitro ischemia and group $\mathrm{B}+$ in vitro ischemia: $\mathrm{P}=0.00$ and $\mathrm{P}=0.01$, respectively) (Fig. 6).

Histological staining. The histological analysis of NP tissues was performed using H\&E staining. Under the highest magnification, the number of NP cells in group $\mathrm{C}$ was significantly increased compared with groups A and B (Fig. 7).

Apotosis analysis of NP tissues was performed using TUNEL staining. The TUNEL-positive NP cells (brown cells) in group $\mathrm{C}$ were markedly reduced compared with groups $\mathrm{A}$ and B (Fig. 7). 
A
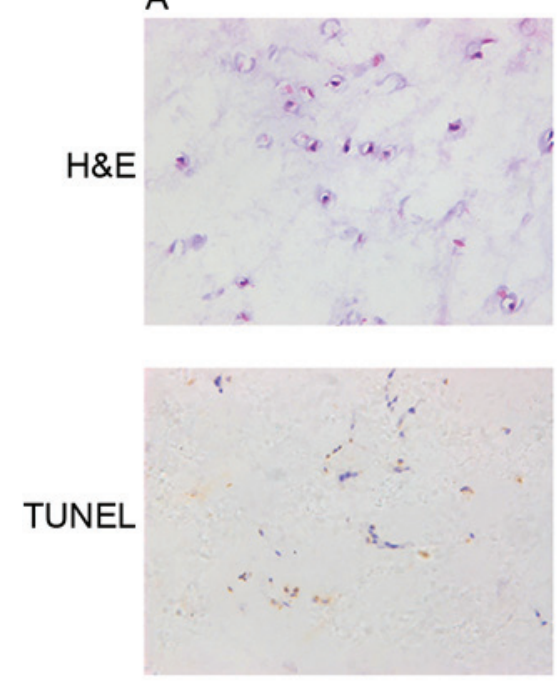

B
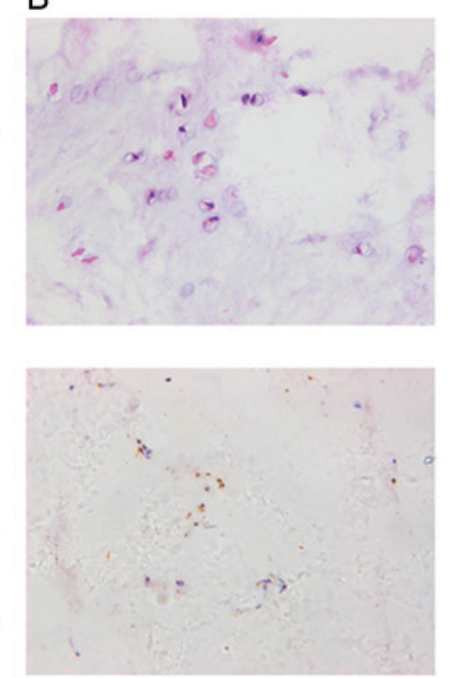

C
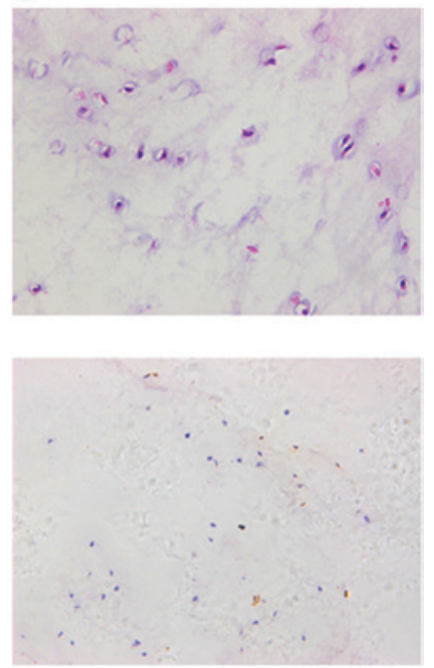

Figure 7. H\&E and TUNEL staining in the different groups. H\&E, hematoxylin and eosin; TUNEL, Terminal deoxynucleotidyl transferase dUTP nick end labeling. A, punctured blank control group; B, punctured empty vector control group; C, treatment group.

\section{Discussion}

The current study demonstrated that $\mathrm{LV}$-survivin injection into the intervertebral discs may slow the process of IDD in vivo. Results from MRI, histology, gene expression, protein content and apoptosis analyses all demonstrated a slowing of the course of injury-induced degeneration.

The degeneration model was established using CT-guided percutaneous needle puncture technology. Although an injury model for disc degeneration does not truly reflect the complex biochemical cascade of human degenerative disc disease, it does suggest that LV-survivin may be a possible treatment method to ameliorate the process of injury-induced degeneration. Similarly, injection surgery was accomplished with CT guidance; however, a microinjector with a needle small enough not to exacerbate further degeneration was used (21). Furthermore, 3 weeks subsequent to the puncture, there was an alteration in the NP visible by MRI, and the degeneration was in an early phase. In the phase, the cellular state might be able to mount a healing response for $\mathrm{LV}$ injection, so this time point was chosen for the LV injection (22).

In the current study, the MRI imaging of all groups at the 0 week time point exhibited normal intervertebral disc morphology. A total of 3 weeks after the puncture, the imaging of all groups demonstrated the same degree of degeneration. A total of 12 weeks after the puncture, treated NPs did not darken and reduce in area as much as the punctured discs. The anatomical morphology of the discs following sacrifice were consistent with that of MRI imaging. These results indicated that LV-survivin injection aided in the preservation of the intervertebral discs.

The gene expression and protein content of survivin in NP tissues of group $\mathrm{C}$ were significantly increased compared with that of groups A and B. These results indicated that there was a stable expression of survivin in NP cells following in vivo LV-survivin injection into the rabbits. Thus, based on the effect and function of survivin, a reduction in caspase-3 activity and apoptosis of NP cells, and an increase in the number of NP cells was detected, compared with the punctured control groups. This observation may elucidate the effect of LV-survivin gene transfection on the deceleration of the degeneration process in vivo.

Caspase- 3 activity was analyzed using the caspase- 3 activity assay, and no alteration between the caspase-3 activities of groups A, B and C was observed in the unstressed NP cells. However, in in vitro ischemiic cultures (1\% oxygen, glucose deprivation), the caspase-3 activity of the NP cells significantly increased compared with unstressed NP cells, and the increase of group C caspase-3 activity was statistically reduced compared with that of groups A and B. These results were consistent with a previous study (17), and indicated that ischemiic culture conditions induced NP cell apoptosis, and that overexpression of survivin may contribute in the reduction of caspase- 3 activity for the anti-apoptotic function in the degeneration process.

In order to identify NP cell numbers and apoptotic alterations occurring in the different groups, $\mathrm{H} \& \mathrm{E}$ staining and TUNEL were used. These results indicated that LV injection with survivin slowed NP cell apoptosis according to the results of caspase- 3 activity, and similarly demonstrated that overexpression of survivin may serve a role under ischemic conditions in vivo or vitro.

Furthermore, in the primary culture of NP cells, no significant different of cell morphology were observed among the three groups. The results were different from the previous studies, with a previous study reporting that in vitro, the morphology of degenerative NP cell subsequent to transfection with LV-survivin was significantly changed (23). The difference may have resulted from the different transfection conditions. The in vitro condition of NP cells is complex, and numerous factors and pathways influence each other, thus the regulation of cell morphology is complicated.

Although the current study identified a number of statistically significant observations, there were limitations. All imaging, gene expression, protein content, apoptosis and histology results demonstrated that the LV injection with survivin had a weak effect on slowing degeneration. 
It is also possible that the 12 -week course of the present study may have been insufficient to demonstrate the full therapeutic effect in the degenerative cascade. This may be due to the fact that in the model used, degeneration has been demonstrated to continue through to a minimum of 24 weeks (24), therefore longer time points may have demonstrated more statistically significant alterations in signal intensity between the treated and punctured discs.

The present study demonstrated, to the best of our knowledge, for the first time that LV injection may slow disc degeneration. Although the results may not necessarily translate to human degenerative disc disease, and the results are preliminary compared with clinical treatment, they provide a potential direction for gene therapy as a treatment for disc degeneration.

\section{Acknowledgements}

The current study was supported by a research grant award from the National Natural Science Foundation of China (grant no. 81371998$)$.

\section{References}

1. Hoy D, Brooks P, Blyth F and Buchbinder R: The Epidemiology of low back pain. Best Pract Res Clin Rheumatol 24: 769-781, 2010.

2. Takahashi K, Aoki Y and Ohtori S: Resolving discogenic pain. Eur Spine J 17 (Suppl 4): S428-S431, 2008.

3. Hillman M, Wright A, Rajaratnam G, Tennant A and Chamberlain MA: Prevalence of low back pain in the community: Implications for service provision in Bradford, UK. J Epidemiol Community Health 50: 347-352, 1996.

4. McMeeken J, Tully E, Stillman B, Nattrass C, Bygott IL and Story I: The experience of back pain in young Australians. Man Ther 6: 213-220, 2001.

5. Fritzell P, Hägg O, Wessberg P and Nordwall A; Swedish Lumbar Spine Study Group: 2001 Volvo award winner in clinical studies: Lumbar fusion versus nonsurgical treatment for chronic low back pain: A multicenter randomized controlled trial from the Swedish Lumbar Spine Study Group. Spine (Phila Pa 1976) 26: 2521-2534; discussion 2532-2534, 2001.

6. Park P, Garton HJ, Gala VC, Hoff JT and McGillicuddy JE: Adjacent segment disease after lumbar or lumbosacral fusion: Review of the literature. Spine (Phila Pa 1976) 29: 1938-1944, 2004.

7. Freemont AJ: The cellular pathobiology of the degenerate intervertebral disc and discogenic back pain. Rheumatology (Oxford) 48: 5-10, 2009.

8. Le Maitre CL, Freemont AJ and Hoyland JA: The role of interleukin-1 in the pathogenesis of human intervertebral disc degeneration. Arthritis Res Ther 7: R732-R745, 2005.
9. Smith LJ, Nerurkar NL, Choi KS, Harfe BD and Elliott DM: Degeneration and regeneration of the intervertebral disc: Lessons from development. Dis Model Mech 4: 31-41, 2011.

10. Rannou F, Lee TS, Zhou RH, Chin J, Lotz JC, Mayoux-Benhamou MA, Barbet JP, Chevrot A and Shyy JY: Intervertebral disc degeneration: The role of the mitochondrial pathway in annulus fibrosus cell apoptosis induced by overload. Am J Pathol 164: 915-924, 2004.

11. Zhao CQ, Liu D, Li H, Jiang LS and Dai LY: Interleukin-1beta enhances the effect of serum deprivation on rat annular cell apoptosis. Apoptosis 12: 2155-2161, 2007.

12. Li F and Ling X: Survivin study: An update of "what is the next wave'? J Cell Physiol 208: 476-486, 2006.

13. Lechler P, Balakrishnan S, Schaumburger J, Grässel S, Baier C, Grifka J, Straub RH and Renkawitz T: The oncofetal gene survivin is re-expressed in osteoarthritis and is required for chondrocyte proliferation in vitro. BMC Musculoskelet Disord 12: 150, 2011.

14. Bokarewa M, Tarkowski A and Magnusson M: Pathological survivin expression links viral infections with pathogenesis of erosive rheumatoid arthritis. Scand J Immunol 66: 192-198, 2007.

15. Yang KS, Yue B and Ma XX: The expression of survivin and its significance in fetal intervertebral disc. Qingdao Daxue Yixueyuan Xuebao 49: 205-206, 2013.

16. Yang KS: The expression of survivin and its significance inintervertebral disc (dissertation) Qingdao University, 2013.

17. Lin, Yazhou Yue, Bin Xiang, Hongfei, et al: Survivin is re-expressed in disc degeneration disease and is required for degenerated nucleus pulposus cell proliferation and anti-apoptosis in vitro. Mol Med Rep (In press).

18. Zhou RP, Zhang ZM, Wang L, Huang MJ, Zheng XC, Cui YN, Yin M, Wang XK, Yao NZ, Chen TY, et al: Establishing a disc degeneration model using computed tomography-guided percutaneous puncture technique in the rabbit. J Surg Res 181: e65-e74, 2013.

19. Livak KJ and Schmittgen TD: Analysis of relative gene expression data using real-time quantitative PCR and the 2(-Delta Delta C(T)) method. Methods 25: 402-408, 2001.

20. Kluba T, Niemeyer T, Gaissmaier C and Gründer T: Human anulus fibrosis and nucleus pulposus cells of the intervertebral disc: Effect of degeneration and culture system on cell phenotype. Spine (Phila Pa 1976) 30: 2743-2748, 2005.

21. Elliott DM, Yerramalli CS, Beckstein JI, Johannessen W and Vresilovic EJ: The effect of relative needle diameter in puncture and sham injection animal models of degeneration. Spine (Phila Pa 1976) 33: 588-596, 2008.

22. Kwon YJ: A minimally invasive rabbit model of progressive and reproducible disc degeneration confirmed by radiology, gene expression, and histology. J Korean Neurosurg Soc 53: 323-330, 2013.

23. Ma X, Lin Y, Yang K, Yue B, Xiang H and Chen B: Effect of lentivirus-mediated survivin transfection on the morphology and apoptosis of nucleus pulposus cells derived from degenerative human disc in vitro. Int J Mol Med 36: 186-194, 2015.

24. Sowa G, Westrick E, Pacek C, Coelho P, Patel D, Vadala G, Georgescu H, Vo N, Studer R and Kang J: In vitro and in vivo testing of a novel regulatory system for gene therapy for intervertebral disc degeneration. Spine 36: E623-E628, 2011. 